\title{
Factors associated with anxiety and depression in rheumatoid arthritis patients: a cross-sectional study
}

\author{
Miyabi Uda ${ }^{1}$ (D, Motomu Hashimoto ${ }^{2,3}$, Ryuji Uozumi ${ }^{4}$, Mie Torii ${ }^{1}$, Takao Fujii ${ }^{5}$, Masao Tanaka², Moritoshi Furu², \\ Hiromu Ito ${ }^{2}$, Chikashi Terao ${ }^{6,7,8,9}$, Wataru Yamamoto ${ }^{10}$, Genichi Sugihara ${ }^{11}$, Yukako Nakagami ${ }^{11,12}$, \\ Tsuneyo Mimori ${ }^{13,14}$ and Kazuko $\mathrm{Nin}^{1 *}$ (i)
}

\begin{abstract}
Background: The management of anxiety and depression symptoms in rheumatoid arthritis (RA) patients is vital. Previous study findings on this topic are conflicting, and the topic remains to be thoroughly investigated. This study aimed to clarify the association of RA disease activity with anxiety and depression symptoms after controlling for physical disability, pain, and medication.

Methods: We conducted a cross-sectional study of RA patients from the Kyoto University Rheumatoid Arthritis Management Alliance cohort. We assessed patients using the Disease Activity Score (DAS28), Health Assessment Questionnaire Disability Index (HAQ-DI), and Hospital Anxiety and Depression Scale (HADS). Anxiety and depression symptoms were defined by a HADS score $\geq 8$. We analyzed the data using multivariable logistic regression analyses.

Results: Of 517 participants, $17.6 \%$ had anxiety symptoms and $27.7 \%$ had depression symptoms. The multivariable logistic regression analysis demonstrated that DAS28 was not independently associated with anxiety symptoms (odds ratio [OR] [95\% confidence interval; $\mathrm{Cl}] 0.93$ [0.48-1.78]; $p=0.82$ ) and depression symptoms (OR [95\% Cl] $1.45[0.81-2.61] ; p=0.22$ ). However, DAS28 patient global assessment (PtGA) severity was associated with anxiety symptoms (OR [95\% Cl] 1.15 [1.02-1.29]; $p=0.03)$ and depression symptoms (OR [95\% Cl] $1.21[1.09-1.35] ; p<0.01)$. Additionally, HAQ-DI scores $>0.5$ were associated with anxiety symptoms (OR [95\% CI] 3.51 [1.85-6.64]; $p<0.01$ ) and depression symptoms (OR [95\% CI] $2.65[1.56-4.50] ; p<0.01)$. Patients using steroids were more likely to have depression than those not using steroids (OR [95\% CI] 1.66 [1.03-2.67]; $p=0.04)$.

Conclusions: No association was found between RA disease activity and anxiety and depression symptoms in the multivariable logistic regression analysis. Patients with high PtGA scores or HAQ-DI-based non-remission were more likely to experience anxiety and depression symptoms, irrespective of disease activity remission status. Rather than focusing solely on controlling disease activity, treatment should focus on improving or preserving physical function and the patient's overall sense of well-being.
\end{abstract}

Keywords: Rheumatoid arthritis, Anxiety, Depression, Patient global assessment (PtGA), Quality of life

*Correspondence: nin@kuhp.kyoto-u.ac.jp

1 Department of Human Health Sciences, Kyoto University Graduate

School of Medicine, 53 Shogoin Kawahara-cho, Sakyo-ku, Kyoto 606-8507,

Japan

Full list of author information is available at the end of the article

\section{Background}

Symptoms of anxiety and depression are more prevalent in rheumatoid arthritis (RA) patients than in the general population $[1,2]$. Studies have shown that $26-46 \%$ of RA patients have anxiety symptoms, and $14.8-34.2 \%$ have 
depression symptoms [1]. RA patients with these conditions have worse outcomes, including poor medication adherence [3], worse response to treatment [3], higher medical costs [4-6], high mortality [7, 8], and lower quality of life (QOL) $[6,9,10]$. Therefore, it is important to examine the risk factors of anxiety and depression symptoms in RA patients and incorporate the management of psychological factors into treatment.

Research has been conducted in this area to improve RA prevention and treatment. However, it is difficult for rheumatologists to observe anxiety and depression symptoms in ambulatory care. In this study, self-report questionnaires such as the Hospital Anxiety and Depression Scale (HADS) [11] were used to assess anxiety and depression symptoms in RA patients. Because of the burden on patients and the time required, it is difficult to administer questionnaires in everyday medical settings. Therefore, we focused on disease activity evaluation during medical examinations. If disease activity and its components correlate with anxiety and depression symptoms in RA patients after excluding other factors that may affect such symptoms, then anxiety and depression could be assessed through disease activity. Thus, the results of disease activity assessment could be used in daily medical care as an index for anxiety and depression symptoms.

RA is an autoimmune inflammatory disease that causes joint deformation and physical dysfunction [12]. Physical disability [13-15] and pain [16-18] are known risk factors for anxiety and depression symptoms in RA patients. Recent studies have demonstrated that these conditions are associated with systemic inflammation caused by proinflammatory cytokines such as tumor necrosis factor- $\alpha$, interleukin-1 $\beta$, and interleukin-6 $[19,20]$. Cytokines are also believed to cause depression by hyperactivation of the hypothalamic-pituitary-adrenal axis [21], and studies have reported that $\mathrm{C}$-reactive protein (CRP) levels are associated with anxiety and depression symptoms in RA patients [22].

Disease activity that reflects the above factors may be associated with anxiety and depression symptoms in RA patients. However, study findings on the correlation between disease activity and anxiety and depression are inconsistent. Some studies have suggested a positive association between RA disease activity and anxiety and depression symptoms $[23,24]$, whereas others have not identified an association [9]. A key reason for this is that other factors associated with anxiety and depression symptoms, such as physical disability, pain, and medication, have not been investigated $[9,23]$.

There are few comparisons of the prevalence of anxiety and depression across diseases with similar progression patterns, and findings are inconsistent. In an early (2009) study, Arne et al. showed that patients with chronic obstructive pulmonary disease had a higher prevalence of depression than patients with RA or diabetes mellitus [25]. A comparison of patients with rheumatic diseases showed that depression was more prevalent in patients with systemic lupus erythematosus and fibromyalgia (34\% and 39\%, respectively) than in those with RA (15\%) [26]. Similarly, a recent study of the disease impact of fibromyalgia, rheumatoid arthritis, spondyloarthritis, and Sjögren's syndrome found a higher prevalence of depression and anxiety in fibromyalgia patients than in RA patients [27]. In contrast, a case control study of depression and insomnia in patients with RA and those with osteoarthritis found that approximately $75 \%$ of RA patients reported depression compared with $50 \%$ of osteoarthritis patients, although the difference was not significant. Both groups of patients also experienced stress-related insomnia [28], suggesting that subjective experiences of stress and well-being may have a greater effect than inflammation on psychological status in RA patients.

We hypothesized that disease activity and its components are associated with anxiety and depression symptoms, even after excluding the effects of pain, disability, and medication on anxiety and depression. Using a rigorous cohort study, we aimed to identify the factors associated with anxiety and depression symptoms in RA patients by simultaneous evaluation of disease activity, pain, physical disability, and medication.

\section{Methods}

\section{Patients and setting}

We performed a cross-sectional analysis of patients who visited the outpatient RA center of Kyoto University Hospital between May 1 and December 31, 2014, and whose data were collected in the 2014 Kyoto University Rheumatoid Arthritis Management Alliance (KURAMA) cohort [29]. All patients fulfilled the 1987 or 2010 American College of Rheumatology and the European League against Rheumatism RA classification criteria [30]. We excluded patients with psychiatric disorders, such as bipolar disorder and schizophrenia. The presence of these disorders was determined by one questionnaire item, which asked patients if they were attending an outpatient appointment in the psychiatry department or in the psychosomatic medicine department; the medical records of psychiatry outpatients were then checked to verify diagnoses of psychiatric disorders. We also excluded patients who did not complete a HADS questionnaire [11]. The study protocol was approved by the Kyoto University Graduate School and Faculty of Medicine, Ethics Committee, and all procedures were performed in accordance with the 1964 Declaration of Helsinki and its later amendments or comparable ethical standards. 


\section{Clinical assessments and outcomes}

RA disease activity was assessed using the Disease Activity Score-CRP (DAS28-CRP), which is based on a 28-joint assessment; 28 tender joint count (TJC), and 28 swollen joint count (SJC); CRP; and the patient global assessment (PtGA) [31, 32] to determine each patient's total RA score. DAS28-CRP-based clinical remission/ non-remission and DAS4 variables (comprising TJC, SJC, CRP, and PtGA) were also used. Clinical remission of disease activity was defined as a DAS28-CRP score $<2.6[31$, 32].

The Health Assessment Questionnaire Disability Index (HAQ-DI) [33] was used to assess physical disability. This is an eight-category questionnaire with 20 subscales that measure functional disability. Each item is scored on a scale of 0-3 (0: without any difficulty; 1 : with some difficulty; 2: with much difficulty; and 3: unable to do), and the average value of the eight categories was calculated. Functional remission was defined as HAQ-DI scores $\leq 0.5$. Pain was evaluated using either the Visual Analogue Scale (VAS), on which items were scored from 0 (no pain) to 100 (maximum pain), or the TJC. Other data, such as age, sex, duration of disease, medication (biological disease-modifying anti-rheumatic drugs [bDMARDs], methotrexate, and steroids), were collected from the KURAMA cohort [29]. Confounding factors included age, sex, pain, HAQ-DI score, and medication use. Age and duration of disease were measured as total scores, and binary sex and medication data were used for the analysis.

The primary study outcomes were anxiety and depression symptoms. The outcomes were evaluated using the HADS [11], a 14-item questionnaire with seven subscales for anxiety and depression symptoms. Each item is scored on a scale of $0-3$, the total score range for each condition is $0-21$. Scores of $0-7$ indicate no or few anxiety or depression symptoms, 8-10 indicate mild anxiety or depression, and $\geq 11$ indicate severe anxiety or depression. The HADS has been widely used in Japan to screen patients with a variety of diseases, and has been previously validated for use with the Japanese population [34]. In this study, we defined anxiety and depression as a HADS anxiety score $\geq 8$ and HADS depression score $\geq 8$, respectively [11].

\section{Statistical analysis}

We followed Peduzzi $[35,36]$ in estimating the required sample size. The logistic model required 10 events per variables and the required sample size $=(10$ cases $/$ variable) $\times$ (number of variables $) \div$ probability of occurrence. The mean probability of occurrence was estimated from rates of anxiety (26-46\%; mean: $36 \%$ ) and depression
(14.8-34.2\%; mean: 24.5\%) previously reported [1]. Using this method, we calculated that the estimated required sample size was 440 .

Unless otherwise stated, the data are presented as the mean and $95 \%$ confidence interval (CI) for normally distributed continuous data, the median and interquartile range for non-normally distributed continuous data, or as frequencies (\%). First, we examined the associations between RA patient characteristics and anxiety and depression symptoms using a univariable logistic regression and calculated the odds ratio (OR) and 95\% confidence interval $(\mathrm{CI})$. We then conducted a multivariable logistic regression to investigate the associations between DAS28-CRP-based remission and anxiety and depression symptoms. Based on clinician judgment and the univariable analysis results, explanatory variables were selected and adjusted for age, HAQ-DI, pain, and medication use (bDMARDs, methotrexate, and steroids). We also conducted a multivariable logistic regression using DAS4 variables scores instead of DAS28-CRP-based remission to determine which factors may separately contribute to anxiety and depression symptoms in RA patients. All analyses were performed using JMP 14.0 (SAS Institute Inc., Cary, NC, USA).

\section{Results}

\section{Patient characteristics}

We assessed 542 RA patients and excluded 25 because they had psychiatric disorders such as bipolar disorder and schizophrenia. Of the remaining 517 patients, 9 were excluded from the anxiety analyses because of incomplete HADS anxiety questionnaires. Additionally, 10 were excluded from the depression analyses because of incomplete HADS depression questionnaires. Finally, 508 patients were included in the final analyses of anxiety symptoms, and 507 patients in the analyses of depression symptoms (Fig. 1). Table 1 shows the patient characteristics. The mean DAS28-CRP score was 1.91 (95\% CI 1.83-1.98), and 360 (78.9\%) patients achieved DAS28CRP-based remission. The mean HAQ-DI score was 0.75 (95\% CI 0.68-0.82), and 252 (51.3\%) patients achieved HAQ-DI-based remission. The mean disease duration was 160.9 (95\% CI 148.8-173.1) months. Of all patients, $91(17.6 \%)$ had anxiety symptoms, and 143 (27.7\%) had depression symptoms as measured by the HADS (Table 1).

\section{Association between clinical variables and anxiety symptoms}

First, we performed a univariable logistic regression. DAS28-CRP-based non-remission, TJC, SJC, CRP, PtGA severity, HAQ-DI-based non-remission, pain severity, and steroid use were selected as possible 


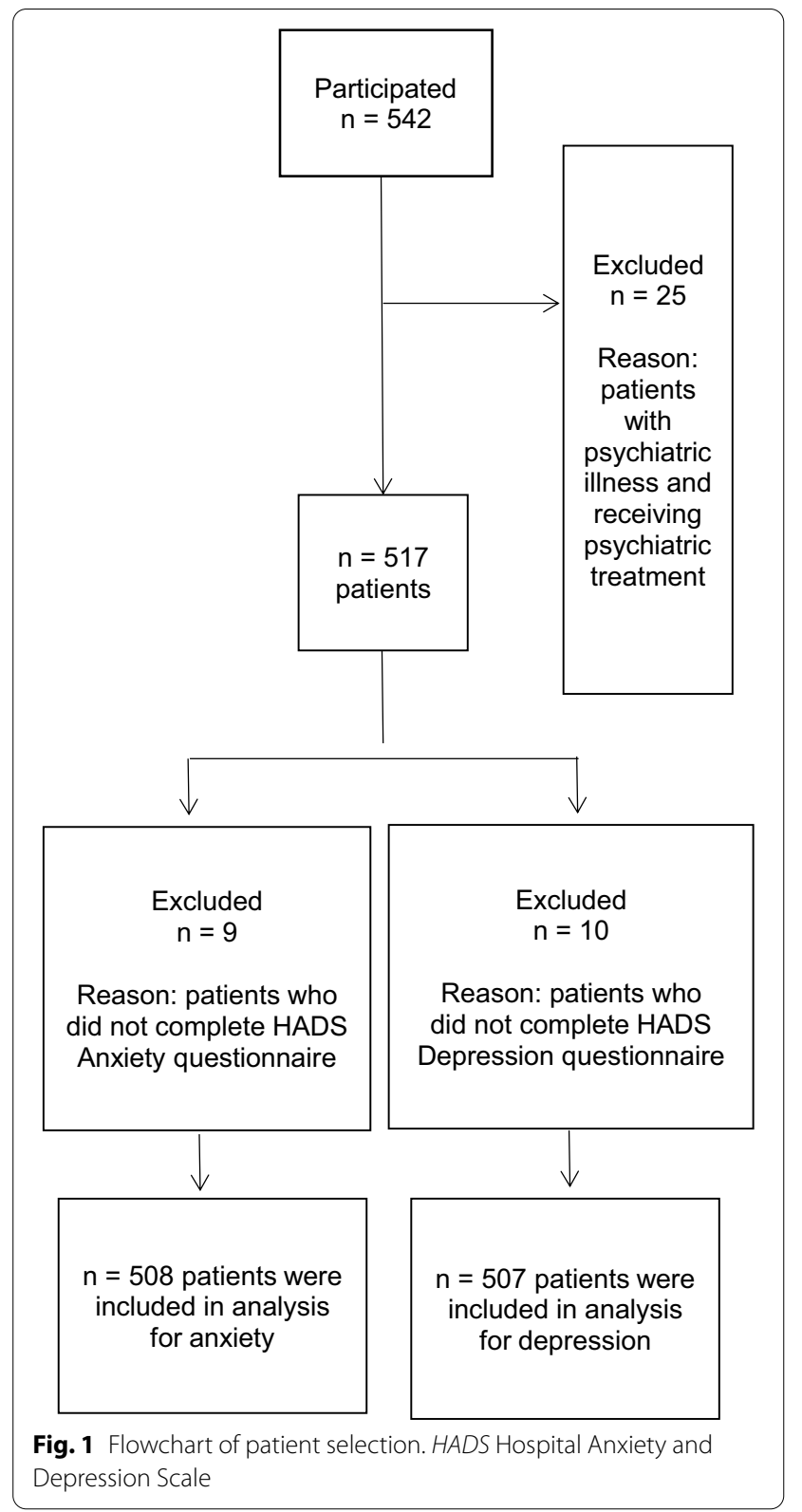

factors associated with anxiety symptoms. We then conducted a multivariable logistic regression (Table 2). In Model 1, patients with DAS28-CRP-based nonremission were no more likely to have anxiety symptoms (OR [95\% CI] 0.93 [0.48-1.78]; $p=0.82$ ). Patients with HAQ-DI-based non-remission were more likely to have anxiety symptoms than those in remission (OR [95\% CI] 3.51 [1.85-6.64]; $p<0.01$ ). Additionally, younger patients tended to have more anxiety symptoms (OR [95\% CI] 0.83 [0.68-1.01]; $p=0.07$ ). In Model 2, pain VAS score was excluded from the
Table 1 Characteristics of patients with rheumatoid arthritis

\begin{tabular}{|c|c|}
\hline Characteristics & $\begin{array}{l}\text { Overall } \\
\mathrm{N}=517\end{array}$ \\
\hline Sex (female) & $429(83.0)$ \\
\hline Age, years & $62.5(61.4-63.6)$ \\
\hline Disease duration, months & $160.9(148.8-173.1)$ \\
\hline \multicolumn{2}{|l|}{ Stage } \\
\hline । & $114(22.1)$ \\
\hline$\|$ & $125(24.2)$ \\
\hline III & $96(18.6)$ \\
\hline IV & $180(35.0)$ \\
\hline \multicolumn{2}{|l|}{ Disease activity } \\
\hline DAS28-CRP & $1.91(1.83-1.98)$ \\
\hline DAS28-CRP remission $^{\dagger}$ & $360(78.9)$ \\
\hline Low disease activity $^{\dagger}$ & $57(12.5)$ \\
\hline Moderate disease activity $^{\dagger}$ & $37(8.1)$ \\
\hline High disease activity $^{\dagger}$ & $2(0.5)$ \\
\hline TJC & $0.82(0.69-0.96)$ \\
\hline SJC & $0.80(0.67-0.93)$ \\
\hline PtGA & $29.1(27.0-31.2)$ \\
\hline CRP & $0.40(0.33-0.47)$ \\
\hline HAQ-DI & $0.75(0.68-0.82)$ \\
\hline HAQ-DI remission & $252(51.3)$ \\
\hline Pain VAS & $27.6(25.4-29.9)$ \\
\hline \multicolumn{2}{|l|}{ Treatment } \\
\hline Biological DMARD & $187(36.2)$ \\
\hline Methotrexate & $345(66.7)$ \\
\hline Steroid & $159(30.8)$ \\
\hline \multicolumn{2}{|l|}{ Anxiety and depression } \\
\hline Anxiety & $91(17.6)$ \\
\hline Depression & $143(27.7)$ \\
\hline
\end{tabular}

Data are presented as mean (95\% confidence interval) or number (\%). Remission: DAS28-CRP <2.6; VAS range: 0 (no pain) to 100 (maximum pain); Anxiety: HADS-A score $\geq 8$, Depression: HADS-D score $\geq 8$

DAS28 Disease Activity Score in 28 joints, TJC tender joint count, SJC swollen joint count, PtGA patient global assessment, CRP C-reactive protein, HAQ-DI Health Assessment Questionnaire Disability Index, HADS-A Hospital Anxiety and Depression Scale-Anxiety subscale, HADS-D Hospital Anxiety and Depression Scale-Depression subscale, DMARD disease-modifying anti-rheumatic drug, VAS Visual Analogue Scale

${ }^{\dagger} \mathrm{N}=456$ for DAS28-CRP remission owing to 61 missing responses

explanatory variables because of collinearity between pain VAS scores and PtGA scores (correlation coefficient, $r=0.78$ ) (Table 3). Furthermore, we used the DAS4 component TJC as a pain variable instead of pain VAS score. Consequently, patients with severe PtGA scores had a notably greater likelihood of anxiety (OR [95\% CI] 1.15 [1.02-1.29]; $p=0.03)$. Similar to Model 1 , Model 2 showed that patients with HAQ-DI-based non-remission were more likely to have anxiety symptoms than those with HAQ-DI-based remission (OR [95\% CI] $3.21[1.69-6.08] ; p<0.01)$. 
Table 2 Multivariate analyses of association between clinical factors and anxiety in patients with rheumatoid arthritis ( $\mathrm{N}=508)$

\begin{tabular}{|c|c|c|c|c|c|c|}
\hline & \multicolumn{2}{|l|}{ Univariable analysis } & \multicolumn{4}{|l|}{ Multivariable analysis } \\
\hline & \multirow[t]{2}{*}{ Odds ratio $(95 \% \mathrm{Cl})$} & \multirow[t]{2}{*}{$\mathrm{p}$ value } & \multicolumn{2}{|l|}{ Model 1} & \multicolumn{2}{|l|}{ Model 2} \\
\hline & & & Odds ratio $(95 \% \mathrm{Cl})$ & $p$ value & Odds ratio $(95 \% \mathrm{Cl})$ & $p$ value \\
\hline Sex & $0.99(0.55,1.89)$ & 0.98 & $0.88(0.45,1.71)$ & 0.71 & $0.83(0.43,1.63)$ & 0.59 \\
\hline Age (10 years) & $1.01(0.84,1.29)$ & 0.98 & $0.83(0.68,1.01)$ & 0.07 & $0.83(0.68,1.01)$ & 0.06 \\
\hline DAS28-CRP non-remission & $1.99(1.12,3.36)$ & 0.01 & $0.93(0.48,1.78)$ & 0.82 & & \\
\hline TJC & $1.18(1.03,1.35)$ & 0.02 & & & $1.02(0.85,1.22)$ & 0.85 \\
\hline SJC & $1.14(0.98,1.34)$ & 0.08 & & & $0.97(0.79,1.19)$ & 0.76 \\
\hline CRP & $1.23(0.90,1.65)$ & 0.17 & & & $1.01(0.70,1.43)$ & 0.98 \\
\hline PtGA (0-10) & $1.29(1.18,1.42)$ & $<0.01$ & & & $1.15(1.02,1.29)$ & 0.03 \\
\hline HAQ-DI non-remission & $4.32(2.59,7.48)$ & $<0.01$ & $3.51(1.85,6.64)$ & $<0.01$ & $3.21(1.69,6.08)$ & $<0.01$ \\
\hline Pain VAS (0-10) & $1.24(1.14,1.35)$ & $<0.01$ & $1.10(0.98,1.23)$ & 0.12 & & \\
\hline Biological DMARD & $1.12(0.69,1.77)$ & 0.65 & $1.07(0.63,1.80)$ & 0.81 & $1.05(0.62,1.77)$ & 0.85 \\
\hline Methotrexate & $0.64(0.40,1.03)$ & 0.06 & $0.67(0.40,1.13)$ & 0.13 & $0.71(0.42,1.20)$ & 0.20 \\
\hline Steroid & $1.63(1.01,2.60)$ & 0.04 & $1.12(0.66,1.92)$ & 0.67 & $1.14(0.66,1.96)$ & 0.64 \\
\hline
\end{tabular}

Model adjusted for age (stratified in 10 decades), pain (VAS), HAQ-DI remission, and treatment type

VAS range: 0 (no pain) to 10 (maximum). Anxiety was measured using the HADS (HADS-A score $\geq 8$ ). DAS28 was used for clinical remission (DAS28 score of <2.6). DAS4 variables comprise TJC, SJC, CRP, and PtGA

Cl confidence interval, DAS28 Disease Activity Score in 28 joints, $T J C$ tender joint count, SJC swollen joint count, PtGA patient global assessment, VAS Visual Analogue Scale, CRP C-reactive protein, HAQ-DI Health Assessment Questionnaire Disability Index, HADS Hospital Anxiety and Depression Scale, DMARD disease-modifying antirheumatic drug

\section{Association between clinical variables and symptoms of depression}

Similar analyses were conducted for depression symptoms. First, we performed a univariable logistic regression. DAS28-CRP-based non-remission, PtGA severity, HAQ-DI-based non-remission, pain severity, and use of methotrexate and steroids were factors identified as potentially associated with depression symptoms. We then conducted a multivariable logistic regression (Table 4). In Model 1, patients with DAS28-CRP-based non-remission were no more likely to have depression symptoms (OR [95\% CI] 1.45 [0.81-2.61]; $p=0.22$ ). Patients with HAQ-DI-based non-remission were more likely to have depression symptoms than those with HAQ-DI-based remission (OR [ [95\% CI] 2.65 [1.56-4.50]; $p<0.01)$. In Model 2, pain VAS score was excluded from the explanatory variables because of collinearity. Furthermore, we used the DAS4 component TJC as a pain variable instead of pain VAS score. Patients with severe PtGA scores were more likely to have depression symptoms (OR [95\% CI] 1.21 [1.091.35]; $p<0.01$ ). Similar to Model 1, Model 2 showed that patients with HAQ-DI-based non-remission were more likely to have depression than those with HAQDI-based remission (OR [95\% CI] 1.95 [1.15-3.31]; $p=0.01)$. Patients taking steroids were more likely to have depression than those not taking steroids (OR [95\% CI] 1.66 [1.03-2.67]; $p=0.04)$.

\section{Discussion}

This was a large-scale cohort study using the KURAMA cohort [29]. We investigated the association of RA disease activity with anxiety and depression symptoms after adjusting for physical disability, pain, and medication in RA patients. We used multiple models to investigate these associations and obtained several notable findings. First, the multivariable logistic regression analysis identified no association between DAS28-based-nonremission and anxiety and depression symptoms. Second, the severity of PtGA, a component of the DAS28 composite measure, was strongly associated with anxiety and depression symptoms, whereas other individual elements of DAS28-CRP (TJC, SJC, and CRP) were not. Third, HAQ-DI-based non-remission was independently and significantly associated with anxiety and depression symptoms. Fourth, patients taking steroids were more likely to have depression symptoms than those not taking steroids.

Findings of previous studies investigating the association between disease activity and anxiety and depression symptoms are inconsistent. Disease activity [23, 24], physical disability [13-15], and pain severity [16-18] are among the factors associated with anxiety and depression in RA patients. However, these studies have two key limitations: (1) DAS28 and pain scores were simultaneously analyzed, but HAQ-DI scores and drug use were not included as covariates [23] and (2) pain, HAQ-DI 


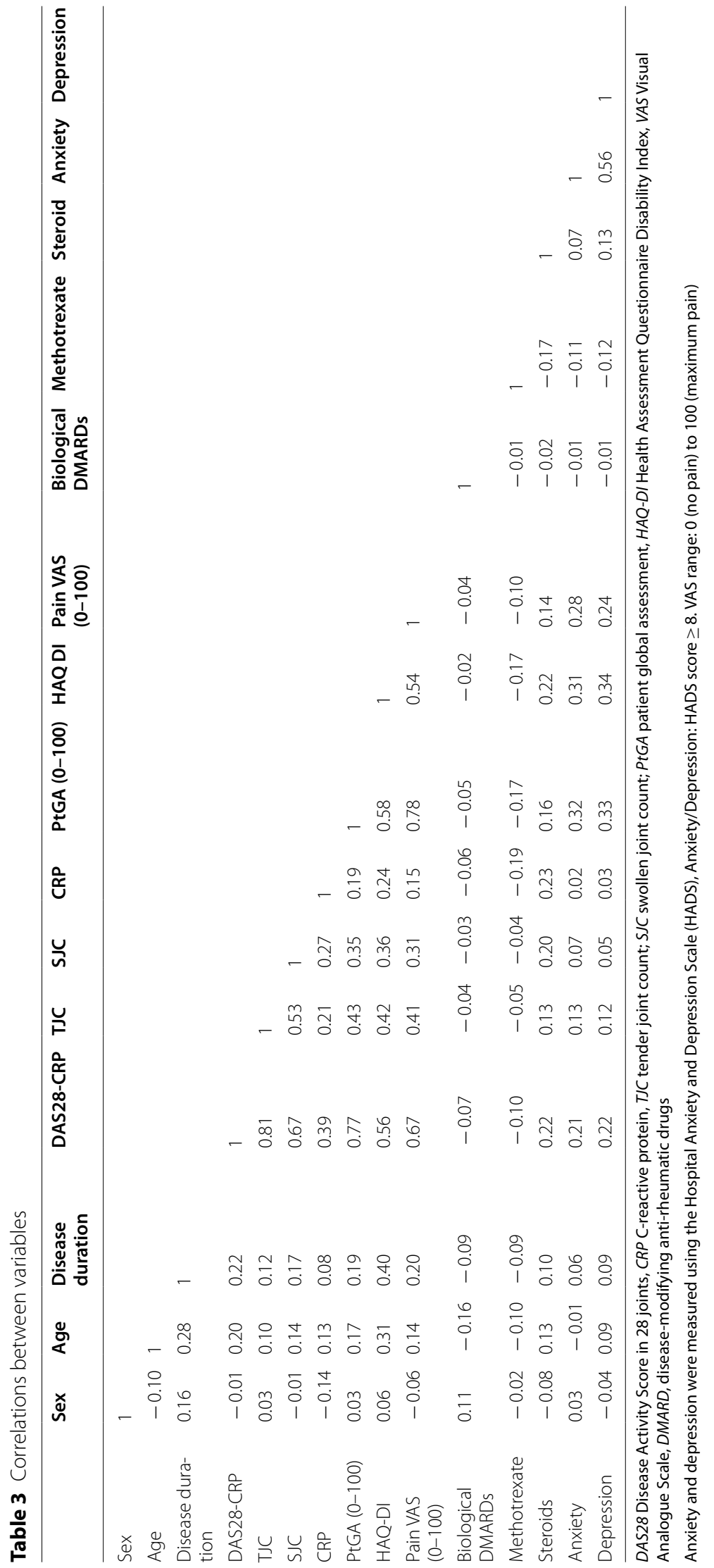


Table 4 Multivariable analyses of association between clinical factors and depression in rheumatoid arthritis patients ( $N=507)$

\begin{tabular}{|c|c|c|c|c|c|c|}
\hline & \multirow{2}{*}{\multicolumn{2}{|c|}{ Univariable analysis }} & \multicolumn{4}{|l|}{ Multivariable analysis } \\
\hline & & & \multicolumn{2}{|l|}{ Model 1} & \multicolumn{2}{|l|}{ Model 2} \\
\hline & Odds ratio $(95 \% \mathrm{Cl})$ & $p$ value & Odds ratio $(95 \% \mathrm{Cl})$ & $p$ value & Odds ratio $(95 \% \mathrm{Cl})$ & $p$ value \\
\hline Sex & $0.80(0.49,1.34)$ & 0.39 & $0.70(0.40,1.22)$ & 0.21 & $0.61(0.34,1.07)$ & 0.09 \\
\hline Age (10 years) & $1.18(1.01,1.38)$ & 0.04 & $1.03(0.87,1.24)$ & 0.70 & $1.04(0.87,1.24)$ & 0.68 \\
\hline DAS28-CRP-based non-remission & $2.37(1.47,3.82)$ & $<0.01$ & $1.45(0.81,2.61)$ & 0.22 & & \\
\hline TJC & $1.10(0.97,1.25)$ & 0.14 & & & $0.96(0.81,1.14)$ & 0.63 \\
\hline SJC & $1.06(0.92,1.21)$ & 0.40 & & & $0.94(0.78,1.14)$ & 0.55 \\
\hline CRP & $1.01(0.74,0.99)$ & 0.96 & & & $0.71(0.50,1.01)$ & 0.06 \\
\hline PtGA (0-10) & $1.27(1.17,1.38)$ & $<0.01$ & & & $1.21(1.09,1.35)$ & $<0.01$ \\
\hline HAQ-DI non-remission & $2.96(1.96,4.53)$ & $<0.01$ & $2.65(1.56,4.50)$ & $<0.01$ & $1.95(1.15,3.31)$ & 0.01 \\
\hline Pain VAS (0-10) & $1.13(1.05,1.22)$ & $<0.01$ & $0.98(0.88,1.09)$ & 0.77 & & \\
\hline Biological DMARDs & $0.92(0.61,1.37)$ & 0.68 & $0.84(0.53,1.33)$ & 0.45 & $0.88(0.55,1.40)$ & 0.58 \\
\hline Methotrexate & $0.60(0.40,0.90)$ & 0.01 & $0.72(0.45,1.14)$ & 0.16 & $0.76(0.48,1.22)$ & 0.26 \\
\hline Steroid & $1.87(1.24,2.81)$ & $<0.01$ & $1.48(0.93,2.34)$ & 0.09 & $1.66(1.03,2.67)$ & 0.04 \\
\hline
\end{tabular}

Model adjusted for age (stratified in 10 decades), pain (VAS), HAQ-DI-based remission, and treatment type. DAS28 score was used to gauge clinical remission (score $<2.6$ ). DAS4 variables comprise TJC, SJC, CRP, and PtGA. VAS range: 0 (no pain) to 10 (maximum pain)

Cl confidence interval, DAS28 Disease Activity Score in 28 joints, CRP C-reactive protein, TJC tender joint count, SJC swollen joint count, PtGA patient global assessment, HAQ-DI Health Assessment Questionnaire Disability Index, VAS Visual Analogue Scale, DMARDs biological disease-modifying anti-rheumatic drugs

scores, and drug use were not included as covariates [9, 24]. Therefore, the association between DAS28 score and anxiety and depression symptoms was mainly evaluated independently, and the effects of other factors may not have been fully evaluated. In this study, we found that when disease activity, disability, pain, and medication were adjusted for, DAS28-based non-remission was not independently associated with anxiety and depression. The average DAS28-CRP score in this study was $1.91 \pm 0.82$, which was lower than scores found in previous studies (DAS28-CRP scores 6.26-6.77). Additionally, almost $80 \%$ of patients achieved remission of disease activity (42.4-56.3\% in previous studies), and disease severity was low and controlled. Therefore, after adjustment, the effects of other factors associated with anxiety and depression symptoms may be more robust than disease activity.

We also found that PtGA (a component of the DAS28CRP composite measure) was notably associated with anxiety and depression symptom, which is similar to findings from a previous study [37]. Moreover, the multivariable analysis results suggested that inflammation (CRP, TJC, and SJC) was not associated with anxiety and depression. Previous studies have reported that systemic inflammation is a potential cause of depression [19-21] and that inflammatory markers correlate with depression [22], suggesting an association between inflammation and depression symptoms. In this study, we did not correlate the two variables because of low CRP. However, PtGA uses the VAS for overall patient self-assessment and is the main tool for measuring patient-reported outcomes [38]. The present results suggest that patient selfassessment, and not inflammation, is associated with anxiety and depression symptoms in RA patients.

PtGA reflects various factors; physical disability [39, 40], pain [18, 39], and catastrophizing pain [41] are among the factors that exacerbate PtGA scores. Santos et al. [42] excluded a priori testing for a direct association between DAS28-CRP3 variables and depression symptoms and instead tested the effects of disease activity and disease impact on patients. They found no direct association between DAS28-CRP3V and depression. They also confirmed an indirect relationship between disease activity and depression symptoms mediated by disease impact, such as pain, physical well-being, functional disability, and coping. Thus, functional disability, pain, recognition, and self-evaluation of disease were reflected in PtGA scores and related to anxiety and depression symptoms. This suggests that individual responses to stress caused by chronic disease, coping mechanisms, and subjective experiences of well-being are at least as important as inflammatory factors in the association between RA and psychological problems [28, 42].

In addition, there is a gap between doctor and patient PtGA assessments [43]. Therefore, to understand patients' anxiety and depression symptoms, it is necessary to pay attention to patients' subjective experience regardless of disease activity. Additionally, to reduce anxiety and depression symptoms in RA patients, it is important to improve patient self-assessment and well-being. 
Cognitive behavioral therapy and mindfulness [44-46] reduce the tendency to catastrophize and subsequently reduce pain and distress; therefore, these approaches may be effective interventions for RA patients.

We found that HAQ-DI score, which identifies physical disability, was associated with anxiety and depression symptoms in a multivariable analysis. Previous studies have found that HAQ-DI score [13-15] is related to anxiety and depression. Regardless of acute disease activity score, functional disability measured by the HAQ-DI is associated with depression [22, 23, 47]. If disability persists, the possibility of concurrent symptoms of anxiety and depression increases, irrespective of disease activity remission. Physical disability reportedly leads to reduced ability to perform household chores and other activities and increased job loss/professional loss [47]. Impairment in the ability to perform daily life activities causes anxiety and depression $[14,47]$.

The effects of bone or joint damage were not clear because joint damage was not evaluated in this study. However, the patients were older and had a longer disease duration, low disease activity, and low inflammation. We suggest that anxiety and depression were caused by loss of activity in daily life owing to prolonged limited function rather than acute symptoms of disease activity. It is important to confirm the effects of the disease on daily life. In the "treat to target" approach, the goal of treatment is to restore physical function so that RA patients can participate in social activities again, thereby maintaining long-term QOL [48]. Improving QOL by supporting daily activities and possibly replacing or recovering lost activities to maintain and improve physical functions may relieve symptoms of anxiety and depression.

Finally, in Model 1, younger RA patients tended to experience anxiety symptoms. Younger RA patients may be more anxious about the future regarding factors such as disease-related concerns, medication, pregnancy, household chores, education, and job-related/ professional work. In Model 2, patients taking steroids were more likely to have depression symptoms. Depression side effects may be caused by the pharmacological actions of steroids [49], so appropriate treatment strategies are needed that consider this risk of side effects.

One of the strengths of this study is that it used a large cohort to investigate the associations between disease activity and anxiety and depression symptoms while adjusting for factors that potentially affect these symptoms. Although a range of scales have been used to evaluate anxiety and depression in RA patients, the HADS was used in this study because scores are unaffected by the physical symptoms associated with the development of RA, such as fatigue and insomnia. Therefore, use of the HADS can help to avoid overestimating anxiety and depression symptoms. The HADS assesses the prevalence of anxiety and depression more accurately than other scales.

Despite our findings, this study had some limitations. First, instead of a formal psychiatric diagnosis, we used self-reported symptoms to determine anxiety and depression levels. Additionally, although we only considered the effects of currently used medication, we could not exclude the effects of prior treatment and drug amounts. Because this study was conducted at a single university hospital, selection bias may also have occurred; most patients had a lengthy disease duration and were in remission or had low disease activity. We also did not collect detailed demographic and socioeconomic data on participants, and therefore the analysis is unable to take such factors into account. Given these limitations, care must be taken in generalizing our findings.

\section{Conclusions}

After adjusting for physical disability, pain, and medication, there was no association between DAS28 and anxiety and depression symptoms. However, patients with high PtGA scores or HAQ-DI-based non-remission were more likely to experience anxiety and depression symptoms. Thus, functional disability, pain, recognition, and self-evaluation of disease were reflected in PtGA and indirectly related to anxiety and depression. To understand anxiety and depression symptoms, it is necessary to pay attention to improve or preserve physical function and patients' subjective experience regardless of disease activity.

\section{Abbreviations}

RA: Rheumatoid arthritis; DAS28: Disease Activity Score; HAQ-DI: Health Assessment Questionnaire Disability Index; OR: Odds ratio; Cl: Confidence interval; PtGA: Patient global assessment; CRP: C-reactive protein; HADS: Hospital Anxiety and Depression Scale; TJC: Tender joint count; SJC: Swollen joint count; VAS: Visual Analogue Scale.

\section{Acknowledgements}

We would like to acknowledge the staff of the Rheumatoid Arthritis Center at the Kyoto University Hospital in Kyoto, Japan, who provided valuable assistance and support in collecting data for this study. We thank Diane Williams, PhD, from Edanz (www.edanz.com/ac) for editing a draft of this manuscript.

\section{Authors' contributions}

$\mathrm{MU}, \mathrm{MH}$, and $\mathrm{KN}$ were responsible for the conception and design of the study. MU, MH, MTorii, TF, MTanaka, MF, HI, CT, and WY acquired data. MU, MH, RU, and KN analyzed and interpreted the data. MU and RU contributed to the statistical analysis. GS, YN, and TM contributed to the interpretation of the data. $\mathrm{MU}, \mathrm{MH}$, and $\mathrm{KN}$ prepared the manuscript. All authors read and approved the final manuscript.

\section{Funding}

The KURAMA cohort study is supported by a grant from Daiichi Sankyo Co., Ltd. This was an investigator-initiated study. The sponsors were not involved 
in the study design; collection, analysis, and interpretation of data; writing of this manuscript; or the decision to submit the manuscript for publication. The authors, their immediate families, and any research foundations with which they are affiliated have not received any financial payments or other benefits from any commercial entity related to the subject of this manuscript.

\section{Availability of data and materials}

All data generated or analyzed during this study are included in this published article or are available from the corresponding author on reasonable request. of Health Information Management, Kurashiki Sweet Hospital, Okayama, Japan. ${ }^{11}$ Department of Psychiatry, Kyoto University Graduate School of Medicine, Kyoto, Japan. ${ }^{12}$ Kyoto University Health Service, Kyoto, Japan. ${ }^{13}$ Department of Rheumatology and Clinical Immunology, Kyoto University Graduate School of Medicine, Kyoto, Japan. ${ }^{14}$ Ijinkai Takeda General Hospital, Kyoto, Japan.

Received: 20 November 2020 Accepted: 17 October 2021

Published online: 29 October 2021

\section{Declarations}

\section{Ethics approval and consent to participate}

The study protocol was approved by the Kyoto University Graduate School and Faculty of Medicine, Ethics Committee, and all procedures were performed in accordance with the 1964 Declaration of Helsinki and its later amendments, or comparable ethical standards. All patients provided written informed consent prior to participating in this study.

\section{Consent for publication}

Publication consent was acquired on the original consent form.

\section{Competing interests}

The Department of Advanced Medicine for Rheumatic Diseases is supported by two local governments in Japan (Nagahama City, Shiga, and Toyooka City, Hyogo) and five pharmaceutical companies (Mitsubishi Tanabe Pharma Corporation, Chugai Pharmaceutical Co., Ltd., AYUMI Pharmaceutical Corporation, Asahi Kasei Corporation, and UCB Japan Co., Ltd.). MH has received grants and/or speaker fees from Bristol-Myers Squibb, Eisai Co., Ltd., Eli Lilly and Company, and Mitsubishi Tanabe Pharma Corporation. RU has received consulting fees from Eisai Co. Ltd., Sawai Pharmaceutical Co. Ltd., and CAC Croit Corporation. TF has received speaking fees and/or honoraria from AbbVie Inc., Astellas Pharma Inc., Asahi Kasei Corporation, Chugai Pharmaceutical Co., Ltd., Eli Lilly and Company, Eisai Co., Ltd., Janssen, Kissei, Mitsubishi Tanabe Pharma Corporation, Ono Pharmaceutical Co., Ltd., Pfizer, Sanofi, Taisho Toyama Pharmaceutical Co., Ltd., Takeda Pharmaceutical Co., Ltd., and UCB Japan Co., Ltd., and has received research grants from AbbVie Inc., AYUMI Pharmaceutical Corporation, Asahi Kasei Corporation, Astellas Pharma Inc., Chugai Pharmaceutical Co., Ltd., Daiichi Sankyo Co., Ltd, Eli Lilly and Company, Eisai Co., Ltd., Kissei, Mitsubishi Tanabe Pharma Corporation, Pfizer, Nippon-Kayaku, Ono Pharmaceutical Co., Ltd., Takeda Pharmaceutical Co., Ltd., and UCB Japan Co., Ltd. MTanaka has received research grants and/or speaker fees from AbbVie GK, Asahi Kasei Pharma Corporation, Astellas Pharma Inc., AYUMI Pharmaceutical Corporation, Bristol-Myers Squibb, Chugai Pharmaceutical Co., Ltd., Eisai Co., Ltd., Eli Lilly Japan K.K., Pfizer Inc., UCB Japan Co., Ltd., Janssen Pharmaceutical K.K., Mitsubishi Tanabe Pharma Corporation, Novartis Pharma K.K., and Taisho Pharma Co., Ltd. $\mathrm{HI}$ has received a research grant and/or speaker fee from Bristol-Myers Squibb, Kyocera, and Asahi Kasei Corporation. TM has received research grants and/or speaker's fees from Asahi Kasei Corporation, Astellas Pharma Inc., AYUMI Pharmaceutical Corporation, Bristol-Myers Squibb, Chugai Pharmaceutical Co., Ltd., Daiichi Sankyo Co., Ltd., Eisai Co., Ltd., Mitsubishi Tanabe Pharma Corporation, Pfizer, and Sanofi. The above companies had no role in the study design, collection or analysis of data, writing of the manuscript, or the decision to submit the manuscript for publication.

\section{Author details}

1 Department of Human Health Sciences, Kyoto University Graduate School of Medicine, 53 Shogoin Kawahara-cho, Sakyo-ku, Kyoto 606-8507, Japan. ${ }^{2}$ Department of Advanced Medicine for Rheumatic Diseases, Kyoto University Graduate School of Medicine, Kyoto, Japan. ${ }^{3}$ Department of Clinical Immunology, Graduate School of Medicine, Osaka City University, Osaka, Japan. ${ }^{4}$ Department of Biomedical Statistics and Bioinformatics, Kyoto University Graduate School of Medicine, Kyoto, Japan. ${ }^{5}$ Department of Rheumatology and Clinical Immunology, Wakayama Medical University, Wakayama, Japan. ${ }^{6}$ Department of Rheumatology and Clinical Immunology, Graduate School of Medicine, Kyoto University, Kyoto, Japan. ${ }^{7}$ Laboratory for Statistical and Translational Genetics, RIKEN Center for Integrative Medical Sciences, Yokohama, Japan. ${ }^{8}$ Clinical Research Center, Shizuoka General Hospital, Shizuoka, Japan. ${ }^{9}$ The Department of Applied Genetics, The School of Pharmaceutical Sciences, University of Shizuoka, Shizuoka, Japan. ${ }^{10}$ Department

\section{References}

1. Matcham F, Rayner L, Steer S, Hotopf M. The prevalence of depression in rheumatoid arthritis: a systematic review and meta-analysis. Rheumatology. 2013;52:2136-48. https://doi.org/10.1093/rheumatology/ket169.

2. Marrie RA, Hitchon CA, Walld R, Patten SB, Bolton JM, Sareen J, et al. Increased burden of psychiatric disorders in rheumatoid arthritis. Arthritis Care Res. 2018;70:970-8. https://doi.org/10.1002/acr.23539.

3. Mattey DL, Dawes PT, Hassell AB, Brownfield A, Packham JC. Effect of psychological distress on continuation of anti-tumor necrosis factor therapy in patients with rheumatoid arthritis. J Rheumatol. 2010;37:2021-4. https://doi.org/10.3899/jrheum.100050.

4. Li X, Gignac MA, Anis AH. The indirect costs of arthritis resulting from unemployment, reduced performance, and occupational changes while at work. Med Care. 2006;44:304-10. https://doi.org/10.1097/01.mlr.00002 04257.25875 .04 .

5. Margaretten M, Julian L, Katz P, Yelin E. Depression in patients with rheumatoid arthritis: description, causes and mechanisms. Int J Clin Rheumatol. 2011;6:617-23. https://doi.org/10.2217/IJR.11.6.

6. Matcham F, Scott IC, Rayner L, Hotopf M, Kingsley GH, Norton S, et al. The impact of rheumatoid arthritis on quality-of-life assessed using the SF-36: a systematic review and meta-analysis. Semin Arthritis Rheum. 2014;44:123-30. https://doi.org/10.1016/j.semarthrit.2014.05.001.

7. Ang DC, Choi H, Kroenke K, Wolfe F. Comorbid depression is an independent risk factor for mortality in patients with rheumatoid arthritis. J Rheumatol. 2005;32:1013-9.

8. Timonen M, Viilo K, Hakko H, Sarkioja T, Ylikulju M, Meyer-Rochow VB, et al. Suicides in persons suffering from rheumatoid arthritis. Rheumatology. 2003;42:287-91. https://doi.org/10.1093/rheumatology/keg082.

9. Ho RC, Fu EH, Chua AN, Cheak AA, Mak A. Clinical and psychosocial factors associated with depression and anxiety in Singaporean patients with rheumatoid arthritis. Int J Rheum Dis. 2011;14:37-47. https://doi.org/10. 1111/j.1756-185X.2010.01591.X.

10. Mikuls T, Saag K, Criswell L, Merlino L, Cerhan JR. Health related quality of life in women with elderly onset rheumatoid arthritis. J Rheumatol. 2003;30:952-7.

11. Zigmond AS, Snaith RP. The Hospital Anxiety and Depression Scale. Acta Psychiatr Scand. 1983;67:361-70. https://doi.org/10.1111/j.1600-0447. 1983.tb09716.x.

12. Cutolo M, Kitas GD, van Riel PL. Burden of disease in treated rheumatoid arthritis patients: going beyond the joint. Semin Arthritis Rheum. 2014;43:479-88. https://doi.org/10.1016/j.semarthrit.2013.08.004.

13. Margaretten $M$, Yelin $E$, Imboden J, Graf J, Barton J, Katz P, et al. Predictors of depression in a multiethnic cohort of patients with rheumatoid arthritis. Arthritis Rheum. 2009;61:1586-91. https://doi.org/10.1002/art.24822.

14. Bacconnier L, Rincheval N, Flipo RM, Goupille P, Daures JP, Boulenger JP, et al. Psychological distress over time in early rheumatoid arthritis: results from a longitudinal study in an early arthritis cohort. Rheumatology. 2015;54:520-7. https://doi.org/10.1093/rheumatology/keu371.

15. Karpouzas GA, Dolatabadi S, Moran R, Li N, Nicassio PM, Weisman MH. Correlates and predictors of disability in vulnerable US Hispanics with rheumatoid arthritis. Arthritis Care Res. 2012;64:1274-81. https://doi.org/ 10.1002/acr.21689.

16. Dickens C, McGowan L, Clark-Carter D, Creed F. Depression in rheumatoid arthritis: a systematic review of the literature with meta-analysis. Psychosom Med. 2002;64:52-60. https://doi.org/10.1097/00006842-20020 1000-00008.

17. Walsh DA, McWilliams DF. Mechanisms, impact and management of pain in rheumatoid arthritis. Nat Rev Rheumatol. 2014;10:581-92. https://doi. org/10.1038/nrrheum.2014.64. 
18. Odegård S, Finset A, Mowinckel P, Kvien TK, Uhlig T. Pain and psychological health status over a 10-year period in patients with recent onset rheumatoid arthritis. Ann Rheum Dis. 2007;66:1195-201. https://doi.org/ 10.1136/ard.2006.064287.

19. de Ridder D, Geenen R, Kuijer R, van Middendorp H. Psychological adjustment to chronic disease. Lancet. 2008;372:246-55. https://doi.org/10. 1016/S0140-6736(08)61078-8.

20. Liu Y, Ho RC, Mak A. Interleukin (IL)-6, tumour necrosis factor alpha (TNFalpha) and soluble interleukin-2 receptors (sIL-2R) are elevated in patients with major depressive disorder: a meta-analysis and meta-regression. J Affect Disord. 2012;139:230-9. https://doi.org/10.1016/j.jad.2011.08.003.

21. Du X, Pang TY. Is dysregulation of the HPA-Axis a core pathophysiology mediating co-morbid depression in neurodegenerative diseases? Front Psychiatry. 2015;6:32. https://doi.org/10.3389/fpsyt.2015.00032.

22. Kojima M, Kojima T, Suzuki S, Oguchi T, Oba M, Tsuchiya H, et al. Depression, inflammation, and pain in patients with rheumatoid arthritis. Arthritis Rheum. 2009;61:1018-24. https://doi.org/10.1002/art.24647.

23. Kekow J, Moots R, Khandker R, Melin J, Freundlich B, Singh A. Improvements in patient-reported outcomes, symptoms of depression and anxiety, and their association with clinical remission among patients with moderate-to-severe active early rheumatoid arthritis. Rheumatology. 2011;50:401-9. https://doi.org/10.1093/rheumatology/keq327.

24. Overman CL, Bossema ER, van Middendorp H, Wijngaards-de Meij L, Verstappen SM, Bulder $M$, et al. The prospective association between psychological distress and disease activity in rheumatoid arthritis: a multilevel regression analysis. Ann Rheum Dis. 2012;71:192-7. https://doi.org/ 10.1136/annrheumdis-2011-200338.

25. Arne M, Janson C, Janson S, Boman G, Lindqvist U, Berne C, et al. Physical activity and quality of life in subjects with chronic disease: chronic obstructive pulmonary disease compared with rheumatoid arthritis and diabetes mellitus. Scand J Prim Health Care. 2009;27:141-7. https://doi. org/10.1080/02813430902808643.

26. Wolfe F, Michaud K, Li T, Latz RS. Chronic conditions and health problems in rheumatic diseases: comparisons with rheumatoid arthritis, noninflammatory rheumatic disorders, systemic lupus erythematosus, and fibromyalgia. J Rheumatol. 2010;37:305-15. https://doi.org/10.3899/jrheum. 090781.

27. Bucourt E, Martaillé V, Goupille P, Joncker-Vannier I, Huttenberger B, Réveillère $C$, et al. A comparative study of fibromyalgia, rheumatoid arthritis, spondyloarthritis, and Sjögren's syndrome; impact of the disease on quality of life, psychological adjustment, and use of coping strategies. Pain Med. 2021;22:372-81. https://doi.org/10.1093/pm/pnz255.

28. Kwiatkowska B, Kłak A, Raciborski F, Maślińska M. The prevalence of depression and insomnia symptoms among patients with rheumatoid arthritis and osteoarthritis in Poland: a case control study. Psychol Health Med. 2019;24:333-43. https://doi.org/10.1080/13548506.2018.1529325.

29. Terao C, Hashimoto M, Yamamoto K, Murakami K, Ohmura K, Nakashima $R$, et al. Three groups in the 28 joints for rheumatoid arthritis synovitisanalysis using more than 17,000 assessments in the KURAMA database. PLoS ONE. 2013;8:e59341. https://doi.org/10.1371/journal.pone.0059341.

30. Aletaha D, Neogi T, Silman AJ, Funovits J, Felson DT, Bingham CO, et al. 2010 rheumatoid arthritis classification criteria: an American College of Rheumatology/European League Against Rheumatism collaborative initiative. Ann Rheum Dis. 2010;69:1580-8. https://doi.org/10.1136/ard. 2010.138461.

31. Wells G, Becker JC, Teng J, Dougados M, Schiff M, Smolen J, et al. Validation of the 28-joint Disease Activity Score (DAS28) and European League Against Rheumatism response criteria based on C-reactive protein against disease progression in patients with rheumatoid arthritis, and comparison with the DAS28 based on erythrocyte sedimentation rate. Ann Rheum Dis. 2009;68:954-60. https://doi.org/10.1136/ard.2007. 084459.

32. Fransen J, Creemers MC, Van Riel PL. Remission in rheumatoid arthritis: agreement of the disease activity score (DAS28) with the ARA preliminary remission criteria. Rheumatology. 2004;43:1252-5. https://doi.org/10. 1093/rheumatology/keh297.

33. Bruce B, Fries JF. The Stanford Health Assessment Questionnaire: a review of its history, issues, progress, and documentation. J Rheumatol. 2003;30:167-78.
34. Kugaya A, Akechi T, Okuyama T, Okamura H, Uchitomi Y. Screening for psychological distress in Japanese cancer patients. Jpn J Clin Oncol. 1998;28:333-8. https://doi.org/10.1093/jjco/28.5.333.

35. Peduzzi P, Concato J, Feinstein AR, Holford TR. Importance of events per independent variable in proportional hazards regression analysis. II Accuracy and precision of regression estimates. J Clin Epidemiol. 1995;48:1503-10. https://doi.org/10.1016/0895-4356(95)00048-8.

36. Peduzzi P, Concato J, Kemper E, Holford TR, Feinstein AR. A simulation study of the number of events per variable in logistic regression analysis. J Clin Epidemiol. 1996;49:1373-9. https://doi.org/10.1016/s0895-4356(96) 00236-3.

37. Katchamart W, Narongroeknawin P, Chanapai W, Thaweeratthakul P, Srisomnuek A. Prevalence of and factors associated with depression and anxiety in patients with rheumatoid arthritis: a multicenter prospective cross-sectional study. Int J Rheum Dis. 2020;23:302-8. https://doi.org/10. 1111/1756-185X.13781.

38. van Tuyl $\mathrm{LH}$, Boers M. Patient-reported outcomes in core domain sets for rheumatic diseases. Nat Rev Rheumatol. 2015;11:705-12. https://doi.org/ 10.1038/nrrheum.2015.116.

39. Khan NA, Spencer HJ, Abda EA, Alten R, Pohl C, Ancuta C, et al. Patient's global assessment of disease activity and patient's assessment of general health for rheumatoid arthritis activity assessment: are they equivalent? Ann Rheum Dis. 2012;71:1942-9. https://doi.org/10.1136/annrh eumdis-2011-201142.

40. Verschueren P, Esselens G, Westhovens R. Predictors of remission, normalized physical function, and changes in the working situation during follow-up of patients with early rheumatoid arthritis: an observational study. Scand J Rheumatol. 2009;38:166-72. https://doi.org/10.1080/03009 740802484846

41. Boyden SD, Hossain IN, Wohlfahrt A, Lee YC. Non-inflammatory causes of pain in patients with rheumatoid arthritis. Curr Rheumatol Rep. 2016;18:30. https://doi.org/10.1007/s11926-016-0581-0.

42. Santos EF, Duarte CM, Ferreira RO, Pinto AM, Geenen R, da Silva JP. Multifactorial explanatory model of depression in patients with rheumatoid arthritis: a structural equation approach. Clin Exp Rheumatol. 2019;37:641-8

43. Barton JL, Imboden J, Graf J, Glidden D, Yelin EH, Schillinger D. Patientphysician discordance in assessments of global disease severity in rheumatoid arthritis. Arthritis Care Res. 2010;62:857-64. https://doi.org/ 10.1002/acr.20132.

44. Beltman MW, Voshaar RC, Speckens AE. Cognitive-behavioral therapy for depression in people with a somatic disease: meta-analysis of randomized controlled trials. Br J Psychiatry. 2010;197:11-9. https://doi.org/10. 1192/bjp.bp.109.064675.

45. DiRenzo D, Crespo-Bosque M, Gould N, Finan P, Nanavati J, Bingham CO Systematic review and meta-analysis: mindfulness-based interventions for rheumatoid arthritis. Curr Rheumatol Rep. 2018;20:75. https://doi.org/ 10.1007/s11926-018-0787-4.

46. Hofmann SG, Gomez AF. Mindfulness-based interventions for anxiety and depression. Psychiatr Clin N Am. 2017:40:739-49. https://doi.org/10. 1016/j.psc.2017.08.008.

47. Katz PP, Yelin EH. Activity loss and the onset of depressive symptoms: do some activities matter more than others? Arthritis Rheum. 2001:44:1194202. https://doi.org/10.1002/1529-0131(200105)44:5\%3c1194::AIDANR203\%3e3.0.CO;2-6.

48. Smolen JS, Aletaha D, Bijlsma JW, Breedveld FC, Boumpas D, Burmester $G$, et al. Treating rheumatoid arthritis to target: recommendations of an international task force. Ann Rheum Dis. 2010;69:631-7. https://doi.org/ 10.1136/ard.2009.123919.

49. Wolkowitz OM, Burke H, Epel ES, Reus VI. Glucocorticoids, mood, memory, and mechanisms. Ann NY Acad Sci. 2009;179:19-40. https://doi.org/10. 1111/j.1749-6632.2009.04980.x.

\section{Publisher's Note}

Springer Nature remains neutral with regard to jurisdictional claims in published maps and institutional affiliations. 